\title{
MicroRNA-491-5p suppresses cervical cancer cell growth by targeting hTERT
}

\author{
QIANG ZHAO ${ }^{1}$, YING-XIAN ZHAI ${ }^{2}$, HUAN-QIU LIU ${ }^{3}$, YING-AI SHI $^{2^{*}}$ and XIN-BAI LI ${ }^{3 *}$ \\ ${ }^{1}$ Department of Pediatric Surgery, The First Hospital, ${ }^{2}$ Key Laboratory of Pathobiology, Ministry of Education, \\ College of Basic Medical Sciences, ${ }^{3}$ Department of Anesthesiology, The First Hospital, \\ Jilin University, Chaoyang, Changchun, Jilin 130021, P.R. China
}

Received January 27, 2015; Accepted April 2, 2015

DOI: $10.3892 / o r .2015 .4013$

\begin{abstract}
MicroRNAs (miRNAs) are small non-coding RNAs that have been shown to regulate a variety of biological processes by targeting messenger RNA. MicroRNA-491-5p (miR-491-5p), an important miRNA, has been demonstrated to be involved in the processes of initiation and progression in several tumors. However, the precise biological function of miR-491-5p and its molecular mechanism in cervical cancer cells remain elusive. The present study was carried out to investigate the clinical significance and prognostic value of miR-491-5p expression in cervical cancer, and to evaluate the role of miR-491-5p and the underlying molecular mechanisms involved in cervical cancer. The results showed that miR491-5p expression was significantly downregulated in cervical cancer tissues when compared with the corresponding adjacent normal tissues $(\mathrm{P}<0.001)$, and the value was negatively associated with advanced International Federation of Gynecology and Obstetrics (FIGO) stage, high histological grading and lymph node metastasis $(\mathrm{P}<0.01)$. The enforced expression of miR-491-5p in cervical cancer cells significantly inhibited proliferation, migration and invasion, induced cell apoptosis, and suppressed the tumor growth of the mouse model of HeLa cells. In addition, the dual-luciferase reporter assay revealed that human telomerase reverse transcriptase (hTERT) was identified as a novel target gene of miR-491-5p. Notably, it
\end{abstract}

Correspondence to: Professor Ying-Ai Shi, Key Laboratory of Pathobiology, Ministry of Education, College of Basic Medical Sciences, Jilin University, 126 Xinmin Street, Chaoyang, Changchun, Jilin 130021, P.R. China

E-mail: shiyingai5312@126.com

Professor Xin-Bai Li, Department of Anesthesiology, The First Hospital, Jilin University, 71 Xinmin Street, Chaoyang, Changchun, Jilin 130021, P.R. China

E-mail: lixinbai126@sina.cn

*Contributed equally

Key words: cervical cancer, microRNAs, miR-491-5p, human telomerase reverse transcriptase was found that miR-491-5p regulated the PI3K/AKT signaling pathway. These results suggested that targeting miR-491-5p is a strategy for blocking the development of cervical cancer.

\section{Introduction}

Cervical cancer is one of the most common malignant tumors of the female reproductive tract, with an estimated global incidence of over 500,000 new cases and 274,000 patients succumbing to the disease annually (1). Radiotherapy, chemotherapy, and surgery have been recently used as the key treatment methods for patients with cervical cancer. However, disease remission results and clinical outcomes remain poor, particularly in patients with advanced stage cervical cancer $(2,3)$. Therefore, understanding of the molecular biology, genetics, causes and cellular origin of cervical cancer to identify potential therapeutic targets for the prevention and treatment of cervical cancer is imperative.

MicroRNAs (miRNAs), which are small non-coding RNA molecules of 21-25 single-stranded nucleotides, are capable of controlling gene expression by inhibiting mRNA translation or by inducing mRNA degradation, predominantly by targeting the 3'-untranslated regions (3'-UTRs) of mRNAs (4). It has been shown that miRNAs play critical roles in the coordination of a wide variety of processes including cell differentiation, proliferation, death and metabolism $(5,6)$. Over 2,500 miRNAs have been identified in the human miRBase database 20.0 (7), and many are involved in tumorigenesis and tumor progression, acting as oncogenes (8) or tumor suppressors (9). In recent years, miRNAs have been identified in the progression of various types of cancer and suggested as novel targets for anticancer therapies and as molecular diagnostic or prognostic markers (10).

miR-491, a recently identified miRNA, is downregulated in several types of cancer, including oral squamous cell carcinoma (OSCC), pancreatic and ovarian cancer, glioblastoma, breast cancer and hepatocellular carcinoma (HCC) (11-16). miR-491-5p, a mature form of miR-491, has been shown to induce apoptosis and inhibit the proliferation of ovarian (13), colorectal (17), pancreatic (12) and breast cancer (16) cells, aand to suppress the migration and invasion of oral squamous cell (11), glioma (18) and breast (19), suggesting that miR-491-5p functions as a 'bona fide' tumor suppressor. 
However, the clinicopathological impact and the exact roles of miR-491-5p and its underlying molecular mechanisms in cervical cancer remain to be identified. In the present study, we investigated the clinicopathological impact of miR-491-5p on cervical cancer patients using quantitative RT-PCR, and the exact roles of miR-491-5p and its underlying molecular mechanisms in cervical cancer cell by several experiments in vitro and in a nude mouse model.

\section{Materials and methods}

Patients and tissue samples. Fresh cervical cancer and matched adjacent normal tissue specimens were collected from 64 patients who underwent surgery between July 2008 and June 2014 in the First Hospital of Jilin University (Jilin, China). The corresponding adjacent normal tissues were obtained beyond $5 \mathrm{~cm}$ away from the boundary of the cervical cancer tissues. The fresh tissue specimens were immediately frozen in liquid nitrogen, and stored at $-80^{\circ} \mathrm{C}$ until use.

Clinicopathological characteristics included patient age, histological grade, tumor size, lymph node metastasis and International Federation of Gynecology and Obstetrics (FIGO) stage (20). The data were collected prospectively. None of the patients recruited in the present study had undergone preoperative chemotherapy or radiotherapy, or other treatment history or other inflammatory diseases. Pathological diagnosis of all 64 cervical cancer patients was cervical squamous cell carcinoma. The study protocol was approved by the Ethics Committee of Jilin University, and the study was conducted according to the principles of the Declaration of Helsinki. Written informed consent was obtained from all of the patients prior to surgery.

Cell culture. Primary normal cervical squamous cells (NCSC) obtained from adjacent non-cancerous cervical tissue were cultured in keratinocyte serum-free medium (Invitrogen-Life Technologies, Carlsbad, CA, USA) supplemented with epithelial growth factor (EGF), bovine pituitary extract and 1\% streptomycin and $1 \%$ penicillin. The MS751, C33A, HeLa, HeLa229 and SiHa cervical cancer cell lines were purchased from the Type Culture Collection of the Chinese Academy of Sciences (Shanghai, China), and were grown in Dulbecco's modified Eagle's medium (DMEM) (Invitrogen) supplemented with $10 \%$ fetal bovine serum (FBS) (HyClone, Logan, UT, USA) and $1 \%$ penicillin/streptomycin (Invitrogen) at $37^{\circ} \mathrm{C}$ in a humidified atmosphere consisting of $5 \% \mathrm{CO}_{2}$.

Quantitative RT-PCR for miR-491-5p. Total RNA was extracted from tissue samples and cells using TRIzol reagent (Invitrogen-Life Technologies, Grand Island, NY, USA) according to the manufacturer's instructions. RNU6B was used as an internal control. Complementary DNA (cDNA) was synthesized from $10 \mathrm{ng}$ of total RNA using the TaqMan miRNA reverse transcription kit (Applied Biosystems, Foster City, CA, USA). Quantitative RT-PCR (RT-qPCR) was performed using the miScript SYBR-Green PCR kit (Qiagen, Hilden, Germany) on an ABI 7900 Real-Time PCR System (Applied Biosystems). RT-qPCR reaction was performed in a final volume of $10 \mu \mathrm{l}$ containing 1X QuantiTect SYBR-Green PCR Master mix (Qiagen), $1 \mu 1$ of the cDNA, and $0.25 \mathrm{mM}$ of each primer (provided by kits). The reaction mixtures were incubated at $95^{\circ} \mathrm{C}$ for $5 \mathrm{~min}$, followed by 40 cycles of $94^{\circ} \mathrm{C}$ for $15 \mathrm{sec}, 56^{\circ} \mathrm{C}$ for $20 \mathrm{sec}$ and $72^{\circ} \mathrm{C}$ for $30 \mathrm{sec}$. The expression levels of miR-491-5p were normalized to the endogenous control RNU6B, and were calculated with the formula $2^{-\Delta \Delta \mathrm{Ct}}$. All the reactions were performed in triplicate.

Cell transfection. miR-491-5p mimic or the corresponding negative control (miR-NC) were produced by the Shanghai GenePharma (Shanghai, China). The cells were transiently transfected using the Lipofectamine 2000 reagent (Invitrogen-Life Technologies) according to the manufacturer's instructions. Transfection efficiencies were evaluated in every experiment by RT-qPCR $24 \mathrm{~h}$ post-transfection.

Cell proliferation. To determine the effect of miR-491-5p on cell proliferation, a CCK-8 assay (Cell Counting kit-8; Dojindo, Japan) was performed. Briefly, $5 \times 10^{3}$ cells were seeded in 96-well plates with $100 \mu \mathrm{l}$ of DMEM and incubated for $24 \mathrm{~h}$. The cells were then transfected with miR-491-5p mimic or miR-NC, respectively, and then cultured for an additional 1-5 days. The proliferative activity was determined at the end of different experimental periods $(24,48,72,96$ and $120 \mathrm{~h}$ ) using the CCK-8 assay according to the manufacturer's instructions.

Colony formation assays. The cells were transfected with miR-491-5p mimics or miR-NC for $48 \mathrm{~h}$. Subsequently, $1 \times 10^{3}$ transfected cells were seeded in 6 -well plates, and cultured for 10 days. Colonies were stained with $1.0 \%$ crystal violet for $1 \mathrm{~min}$ after fixation with $10 \%$ formaldehyde for $10 \mathrm{~min}$. The percentage of colony formation was calculated by adjusting control cells to $100 \%$.

Cell apoptosis assay. The percentage of apoptotic cells was assessed by the TUNEL method. Briefly, the cells were transfected with miR-491-5p mimic or miR-NC for $48 \mathrm{~h}$, respectively. Apoptotic cells were then determined using an In Situ Cell Death Detection kit (POD; Roche Diagnostics, Branchburg, NJ, USA) according to the manufacturer's instructions. The number of apoptotic cells were counted and averaged from three visual fields.

In addition, we detected $\mathrm{Bcl}-2$ and XIAP protein expression by western blotting in cells $24 \mathrm{~h}$ after transfection with miR-491-5p mimic or miR-NC as an additional indicator of apoptosis.

Cell migration and invasion assays. Cell invasion and migration assays were performed using Transwell inserts (Corning, Corning, NY, USA) according to the manufacturer's instructions. For the invasion assay, filters were precoated with Matrigel (BD Biosciences, Bedford, MA, USA) for $30 \mathrm{~min}$. Approximately $3 \times 10^{4}$ transfected cells were placed in the upper chamber in serum-free DMEM medium. The lower chamber of the Transwells was filled with the DMEM medium containing $20 \%$ FBS. After $48 \mathrm{~h}$, the cells on the upper side of the filters were mechanically removed by wiping with a cotton swab. Cells that migrated and invaded on the lower surface of the filter were fixed with $4 \%$ paraformaldehyde for $10 \mathrm{~min}$ and stained with $0.1 \%$ crystal violet for $5 \mathrm{~min}$. The invading 
or migrating cells were photographed under a phase-contrast microscope (Olympus, Tokyo, Japan) and counted in five randomly chosen fields.

miRNA target predictions. Prediction of miR-491-5p targets was performed according to the algorithms: Targetscan 5.1, http://www.targetscan.org; Diana, http://www.diana.cslab.ece. ntua.gr and miRanda, http://www.microrna.org.

Vector construction and luciferase reporter assay. The human hTERT 3'-UTR oligonucleotides containing the wild-type (WT) or mutant (MT) miR-491-5p binding site were cloned into the pGL3-control vector (Ambion, Austin, TX, USA) at the NheI and XhoI sites. HeLa cells $\left(3 \times 10^{4}\right)$ were seeded in triplicate in 24-well plates and allowed to settle for $24 \mathrm{~h}$. The samples were transiently co-transfected with the luciferase reporter plasmid (wt/mt) and either miR-491-5p mimic or miR-NC using Lipofectamine 2000 according to the manufacturer's instructions. Luciferase and Renilla activity were measured $48 \mathrm{~h}$ after transfection using the Dual-Luciferase Reporter Assay kit (Promega, Madison, WI, USA) according to the manufacturer's instructions. The specific activity was expressed as the fold-changes of the experimental versus the control group.

Western blotting. Protein was extracted from tissues and cells using RIPA lysis buffer containing proteinase inhibitor (Sigma). After $30 \mathrm{~min}$ on ice, lysates were clarified by centrifugation at $13,000 \mathrm{x} \mathrm{g}$ at $4^{\circ} \mathrm{C}$ for $10 \mathrm{~min}$, and concentrations of total cellular protein were determined using the Bradford assay (Bio-Rad, Marnes-la-Coquette, France). An qual amount of proteins $(20 \mu \mathrm{g})$ was separated by sodium dodecylsulfate-polyacrylamide gels (SDS-PAGE) on $8-15 \%$ gradient and transferred to polyvinylidene fluoride (PVDF) membranes (Millipore, Billerica, MA, USA) using the Trans-Blot Turbo Transfer system (Bio-Rad). The membranes were blocked with 5\% non-fat dry milk for $2 \mathrm{~h}$ and incubated with primary antibody overnight at $-4^{\circ} \mathrm{C}$ as follows: anti-Bcl-2 $(1: 1,000)$, anti-XIAP $(1: 2,500)$ (both from Abcam, Cambridge, UK), antiGAPDH (1:2,000; Cell Signaling Technology, New England Biolabs); anti-MMP-2 (1:2,000); anti-MMP-9 (1:4,000); antihTERT $(1: 1,000)$; anti-PI3K (1:2,000); anti-phosphorylated (p)-PI3K (Tyr458, 1:1500); anti-AKT (1:1,000) and anti-p-AKT (Ser473; 1:500) (all from Cell Signaling Technology). The membranes were incubated with horseradish peroxidase-conjugated secondary antibodies (Cell Signaling Technology) for $1 \mathrm{~h}$ at room temperature. The immune complexes were detected by enhanced chemiluminescence (Cell Signaling Technology). GAPDH was used to normalize for protein loading. In some cases, the protein expression was measured by quantifying the density of immunoblot bands adjusted to GAPDH using image analysis software ImageJ (Bio-Rad).

Tumor growth in xenograft models. To investigate the effects of miR-491-5p on the tumorigenicity of the xenograft and the influence on survival of tumor-burdened animals, 30 female BALB/c nude mice (aged 4-6 weeks) were obtained from the Tonghua Laboratory Animal Center of Beijing, China, and maintained under specific pathogen-free conditions at the Laboratory Animal Center of Jilin University. Animal experiments were performed in accordance with the institutional guidelines, following a protocol approved by the Ethics Committee of the Jilin University.

Approximately $2.5 \times 10^{6}$ logarithmically growing untreated HeLa cells, stable expressing miR-491 mimic or miR-NC cells suspended in $100 \mu$ l of PBS containing 10\% Matrigel were injected into the flanks of mice $(n=10)$, respectively. Tumor volume was continuously blindly measured by periodic caliper every five days until the mice were sacrificed under anesthesia. The volume was calculated using the formula: $(\pi / 6 \mathrm{x}$ length $\mathrm{x}$ width $\mathrm{x}$ height). Each tumor was excised and weighed when mice were sacrificed on day 30. Parts of each tumor tissue were used to determine cell apoptosis in vivo by TUNEL.

Statistical analysis. Data are presented as the means \pm standard deviation (SD), and the experiments of in vitro were repeated at least three times. A Student's t-test and a one-way analysis of variance were used to assess significant differences between groups. GraphPad Prism version 5.01 (GraphPad Software, San Diego, CA, USA) was used for the statistical analysis. $P<0.05$ was considered to indicate a statistically significant result.

\section{Results}

miR-491-5p downregulation in human cervical cancer is associated with the clinicopathological characteristics of cervical cancer patients. miR-491-5p expression was detected in 64 pairs of cervical cancer and adjacent normal tissues by RT-qPCR. As shown in Fig. 1A, following the normalization to RNU6B expression levels, the miR-491-5p expression level in cervical cancer tissues $(0.41 \pm 0.03)$ was significantly lower than that of the corresponding adjacent normal tissues $(0.79 \pm 0.04)$ $(\mathrm{P}<0.001)$. The median expression level of miR-491-5p (0.41) was used as a cut-off point to divide the 64 patients into two groups: cervical cancer patients expressing miR-491-5p at levels less than the cut-off value were assigned to the low expression group $(n=30)$, while those with a miR-491-5p expression higher than the cut-off value were assigned to the high expression group $(n=34)$. In addition, the association between miR-491-5p expression and clinicopathological characteristics in cervical cancer were investigated. It was found that miR-491-5p expression was significantly lower in the cancer tissues of patients with advanced FIGO stage cervical cancer, lymph node metastasis-positive patients, and poorly differentiated tumors as compared to those of patients with early FIGO stage, lymph node metastasis-negative patients, and well or moderately differentiated tumors, respectively (Table I). No correlation was found between miR-491-5p expression and age, and tumor size. These data suggested that miR-491-5p is involved in the initiation and progression of cervical cancer.

miR-491-5p expression levels in MS751, C33A, HeLa, HeLa229 and SiHa cervical cancer lines and primary NCSC were examined by RT-qPCR. The miR-491-5p expression level was lower in all five cervical cancer cell lines as compared to that of NCSC (Fig. 1B). The HeLa cell line, which exhibited the lowest levels of miR-491-5p expression in the five cell lines, was selected for subsequent experiments.

miR-491-5p inhibits proliferation and colony formation, and induces apoptosis in ovarian cancer cells. To analyze the effect 

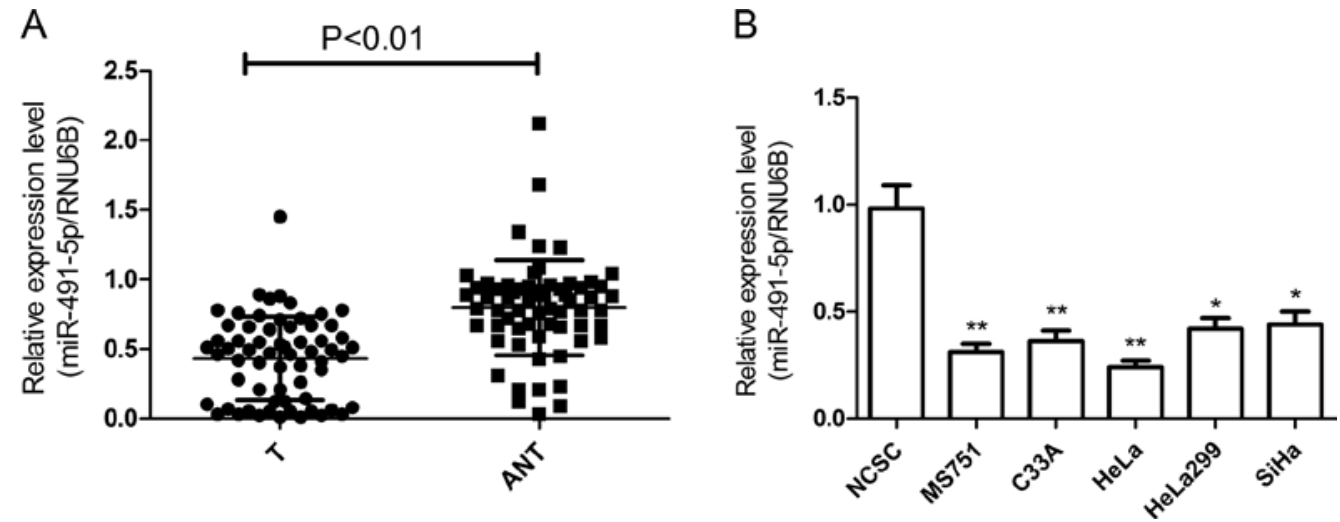

Figure 1. miR-491-5p expression is decreased in cervical cancer tissues and cell lines. (A) Expression of miR-491-5p in 64 paired cervical tumor tissues (T) and their adjacent normal tissues (ANT) by RT-qPCR. miR-491-5p expression was normalized by RNU6B expression. (B) Expression of miR-491-5p in normal cervical squamous cells (NCSCs) and cervical cancer cell lines (MS751, C33A, HeLa, HeLa229 and SiHa) by RT-qPCR. "P<0.05, "P<0.01 vs. NCSCs.

Table I. Association between miR-491-5p expression and clinicopathological characteristics of human cervical cancer.

\begin{tabular}{lcccc}
\hline & & \multicolumn{2}{c}{ miR-491-5p expression } & \\
\cline { 3 - 4 } Variables & $\begin{array}{c}\text { No. of } \\
\text { cases }\end{array}$ & $\begin{array}{c}\text { Low } \\
(\mathrm{n} \%)\end{array}$ & $\begin{array}{c}\text { High } \\
(\mathrm{n} \%)\end{array}$ & P-value \\
\hline Age (years) & & & & 0.489 \\
$<50$ & 28 & $13(46.4)$ & $15(53.6)$ & \\
$\geq 50$ & 36 & $17(47.2)$ & $19(52.8)$ & \\
Tumor size & & & & 0.547 \\
$<5$ & 25 & $12(48.0)$ & $13(52.0)$ & \\
$\geq 5$ & 39 & $18(46.2)$ & $21(53.8)$ & \\
FIGO stage & & & & $<0.01$ \\
Ib-III & 40 & $10(25.0)$ & $30(75.0)$ & \\
IIb-IIIa & 24 & $20(83.3)$ & $4(16.7)$ & \\
Histological grades & & & & $<0.01$ \\
Well/moderate & 38 & $7(18.4)$ & $31(81.6)$ & \\
Poor & 26 & $23(88.4)$ & $3(11.6)$ & \\
Lymph node & & & & $<0.01$ \\
metastasis & & & & \\
No & 45 & $12(26.7)$ & $33(73.3)$ & \\
Yes & 19 & $18(94.7)$ & $1(5.3)$ & \\
\hline
\end{tabular}

FIGO, Federation of Gynecology and Obstetrics.

of miR-491-5p on proliferation of cervical cancer cells, we transfected miR-491-5p mimics into HeLa cells, and a CCK-8 assay was performed. RT-qPCR analysis confirmed that transfected miR-491-5p mimics induced miR-491-5p expression upregulation (Fig. 2A). The CCK-8 assays showed that the overexpression of miR-491-5p markedly inhibited cell proliferation compared to the miR-NC and control groups (Fig. 2B).

Colony formation was determined to assess the role of miR-491-5p in cervical cancer cell growth. The number of HeLa colonies was significantly reduced by th eoverexpression of miR-491-5p (Fig. 2C).
As miR-491-5p significantly affected cell proliferation in HeLa cells, we hypothesized that miR-491-5p was able to function by affecting the cell apoptosis of cervical cancer cells. A TUNEL assay was performed and the results showed that overexpression of miR-491-5p significantly induced cell apoptosis compared to the miR-NC and control groups (Fig. 2D).

To determine the potential mechanism of cell apoptosis in vitro, the anti-apoptosis $\mathrm{Bcl}-2$ and XIAP protein expression was detected in HeLa cells following transfection with miR-491-5p mimics. Western blotting revealed that Bcl-2 and XIAP expression was decreased in miR-491-5p treatment group compared to the control and miR-NC groups (Fig. 2E).

miR-491-5p inhibits migration and invasion in ovarian cancer cells. To identify the role of miR-491-5p in cervical cancer cell metastasis, cell migration and invasion were determined in HeLa cells following transfection with miR-491-5p mimics by the Transwell assay. The cells treated with miR-491-5p mimics significantly decreased the migratory and invasive potential (Fig. 3A and B). These results showed that miR-491-5p is involved in the metastasis of cervical cancer cells.

MMP-2 and -9 are known to play important roles in the metastasis of cervical cancer $(21,22)$. We determined MMP-2 and -9 expression in cervical cancer cells following transfection with miR-491-5p mimics. The western blot analysis revealed that the overexpression of miR-491 decreased the expression of MMP-2 and -9 protein expression in cervical cancer cells (Fig. 3C). These results suggested that miR-491-5p inhibited the cell migration and invasion of cervical cancer cells, at least in part by regulating MMP- 2 and -9 expression.

hTERT are direct targets of miR-491-5p in cervical cancer cells. To identify the target genes of miR-491-5p regulation, we searched publically available databases and selected hTERT as potential downstream target genes (Fig. 4A). As predicted, the western blot analysis revealed that the overexpression of miR-491-5p in HeLa cells decreased the expression of hTERT proteins (Fig. 4B). The dual-luciferase reporter assay showed that when miR-491-5p was overexpressed, the luciferase activity in the reporter vector bearing the wild-type hTERT 3'-UTR fragment was significantly reduced (Fig. 4C), while the activity in the reporter vector with the mutated hTERT 
A
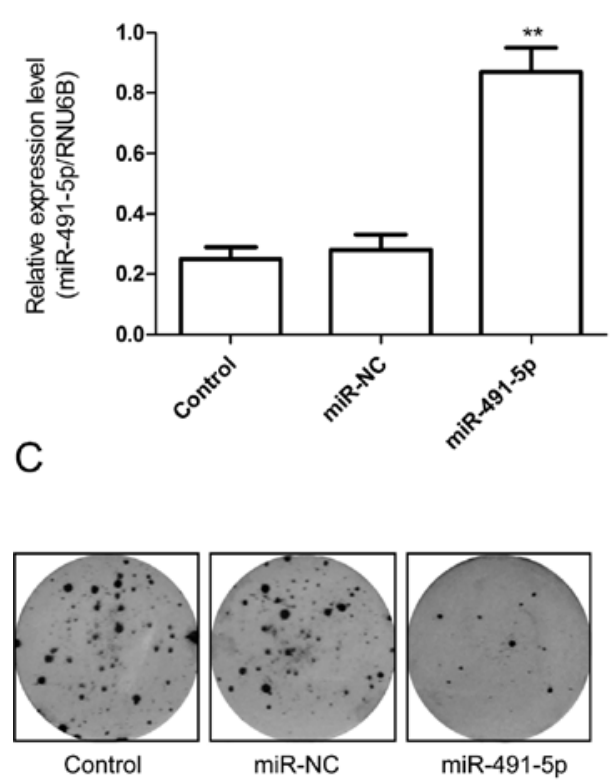

B
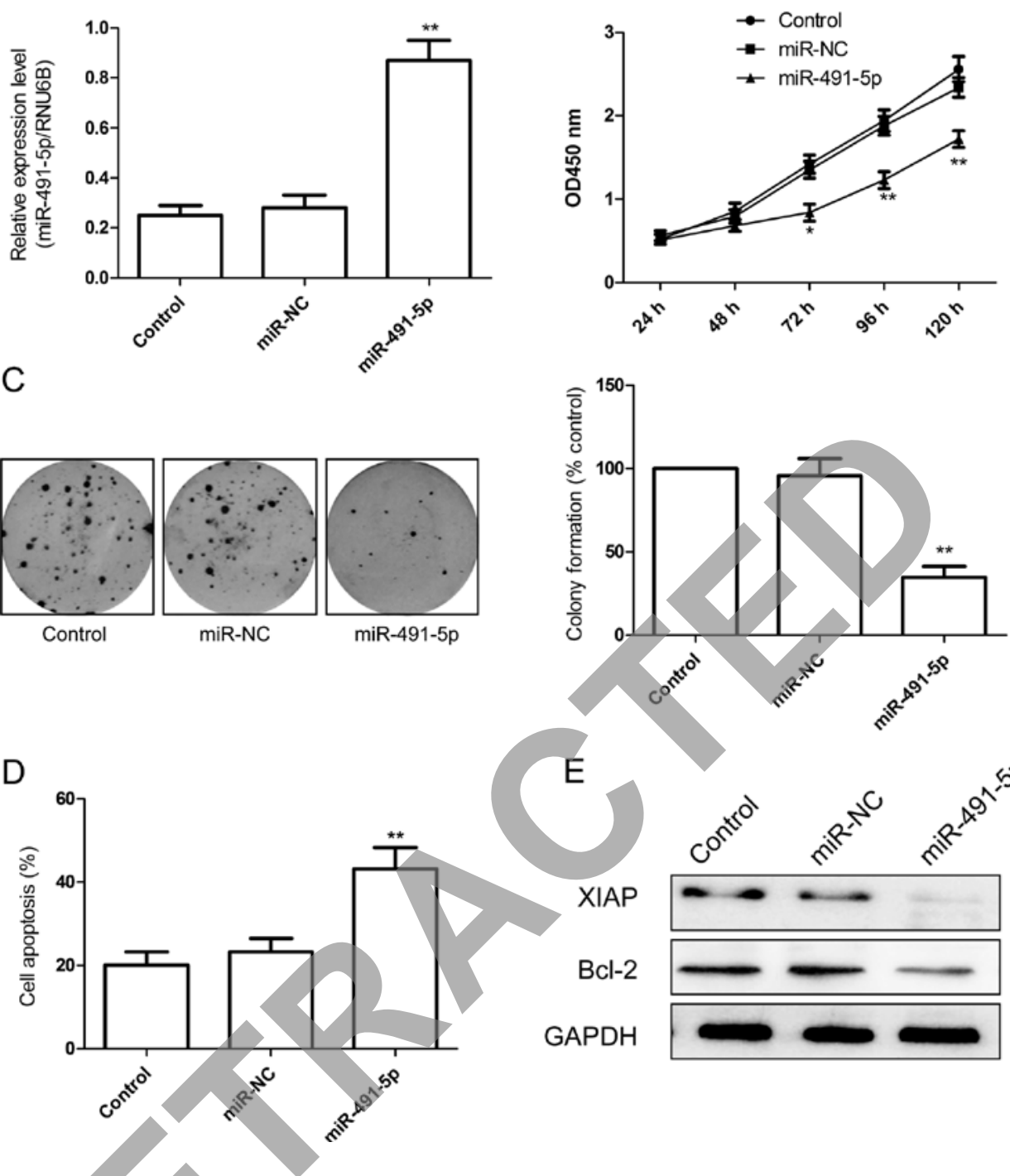

Figure 2. miR-491-5p upregulation innhibits proliferation, colony formation, and induces apoptosis of cervical cancer cells. (A) Expression of miR-491-5p in HeLa cells transfected with miR-491 mimics was detected by RT-qPCR. (B) Effects of miR-491-5p overexpression on the proliferation of cervical cancer cells were analyzed by a CCK-8 assay. (C) Effects of miR-491-5p overexpression on the colony formation of cervical cancer cells were determined. (D) Effects of miR-491-5p overexpression on the apoptosis of cervical cancer cells were determined by TUNEL. (E) The Bcl-2 and XIAP protein expression in cervical cancer cells transfected with miR-491-5p mimic were determined by western blotting. " $\mathrm{P}<0.05,{ }^{\text {" }} \mathrm{P}<0.01$ vs. miR-NC.

3'-UTR was not affected by miR-491-5p. These results indicated that miR-491-5b negatively regulates hTERT expression by directly binding to a unique sequence in the 3'-UTR of hTERT mRNA.

miR-491-5p regulates the PI3K/AKT signaling pathway. The $\mathrm{PI} 3 \mathrm{~K} / \mathrm{AKT}$ pathway is involved in cell proliferation and migration, and invasion. Notably, our previous study showed that the downregulated expression of hTERT inhibited activation of the PI3K/AKT signaling pathway (23). Therefore, we hypothesized that miR-491-5p regulated the PI3K/AKT signaling pathway. We detected the PI3K, p-PI3K, AKT and p-AKT expression in cervical cells after transfection with miR-338-3p mimic or miR-NC by western blotting. The results showed that miR-491-5p reduced the p-PI3K (Tyr458) p-AKT (Serine473) relative to the miR-NC and control groups, whereas the total PI3K and AKT remained unchanged in each group (Fig. 5).
miR-491-5p suppresses cervical cancer xenograft growth. In vitro studies have shown that miR-491-5p is frequently decreased in cervical cancer and is important in cell survival in vitro. We examined the effects of miR-491-5p on tumor growth using a HeLa cervical cancer xenograft model. As shown in Fig. 6A, tumors continued growing in the miR-NC and control groups. However, miR-491-5p significantly reduced tumor growth $(\mathrm{P}<0.05)$. At the end of treatment, the mice were sacrificed, and tumor tissues were cut into strips and the weights were measured. It was found that tumor tissue weight was significantly lower in the miR-491-5p group than those of the miR-NC and control groups (Fig. 6B).

In addition, cell apoptosis of tumor tissue was determined by TUNEL. It was found that the cell apoptotic ratio of miR-491-5p were significantly higher than that of the miR-NC and control groups $(\mathrm{P}<0.05$, Fig. $6 \mathrm{C})$. These results demonstrated that miR491-5p suppressed the tumor growth of cervical cancer in vivo. 
A
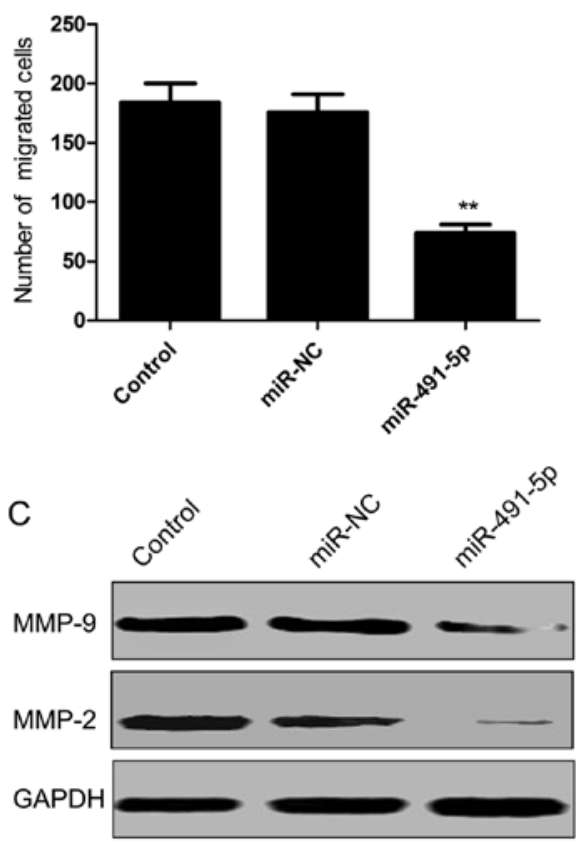

B
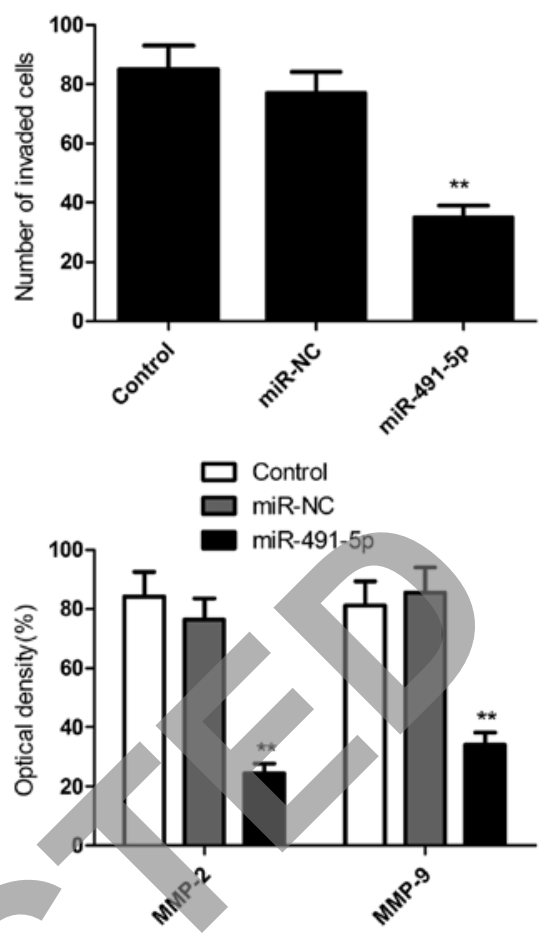

Figure 3. miR-491-5p upregulation inhibits the migration and invasion of cervical cancer cells. (A) The number of migrated cells was determined using a Transwell assay (without Matrigel) in HeLa cells following transfection with miR-491-5p mimics. (B) The number of invaded cells was determined using a Transwell matrix penetration assay (with Matrigel) in HeLa cells following transfection with miR-491-5p mimics. (C) The MMP-2 and -9 protein expression in cervical cancer cells transfected with miR-491-5p mimic was determined by western blotting. The expression levels of MMP-2, MMP-9 and GAPDH (loading control) were determined by densitometry. ${ }^{*} \mathrm{P}<0.05,{ }^{*} \mathrm{P}<0.01$ vs. miR-NC.

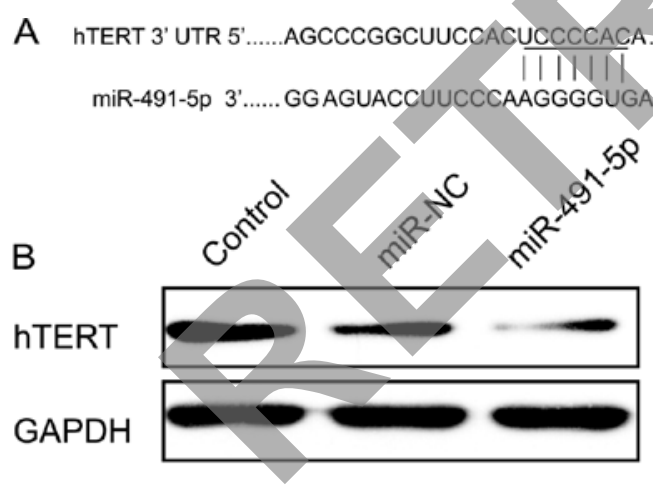

C

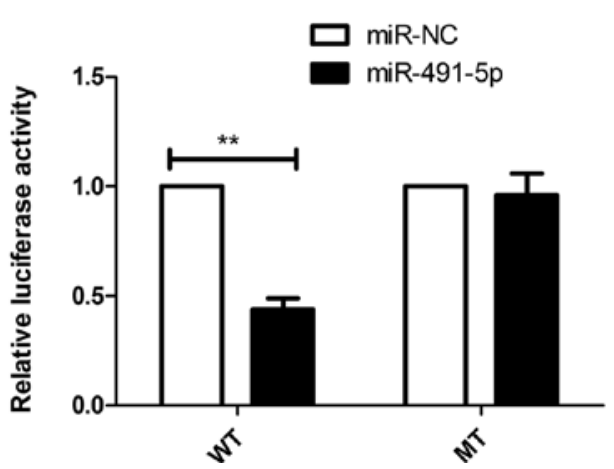

Figure 4. hTERT is directly targeted by miR-491-5p. (A) Predicted miR491-5p target sequences in the 3'-UTRs of hTERT genes. (B) Western blot analysis of hTERT protein expression in HeLa cells following transfection with miR-491-5p mimics. (C) The dual-luciferase reporter assay was performed to confirm the direct regulation of miR-491-5p on hTERT 3'-UTR. ${ }^{*} \mathrm{P}<0.05,{ }^{*} \mathrm{P}<0.01$ vs. miR-NC. hTERT, human telomerase reverse transcriptase; 3'-UTRs, 3'-untranslated regions.

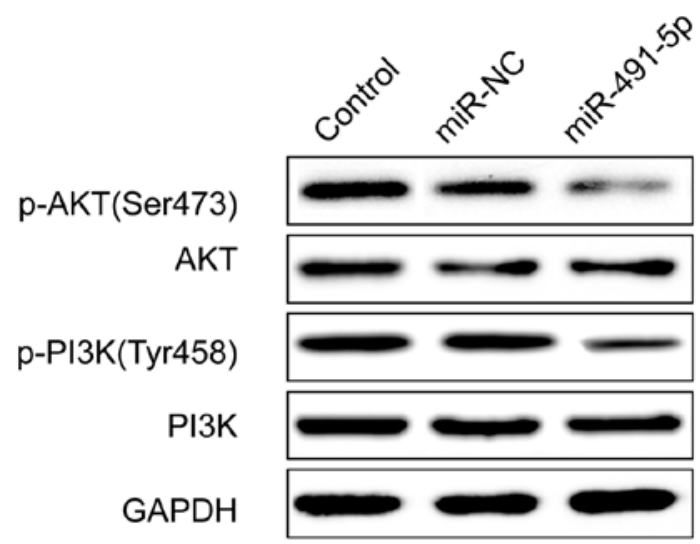

Figure 5. miR-491-5p inhibits PI3K/AKT signaling pathway in cervical cancer cells. Western blot analysis was performed using specific antibodies against the indicated proteins in HeLa cells following transfection with miR-491 mimics. Blots were reprobed for GAPDH to normalize each lane for protein content.

\section{Discussion}

Cervical cancer is one of the common malignancies among women worldwide (1). Despite the comprehensive therapy of surgical resection combined with chemo- and radiotherapy, the median survival has not yet been improved (3). Therefore, the development of novel therapeutic approaches by targeting the molecules that are altered in this disease is crucial to improve 
A

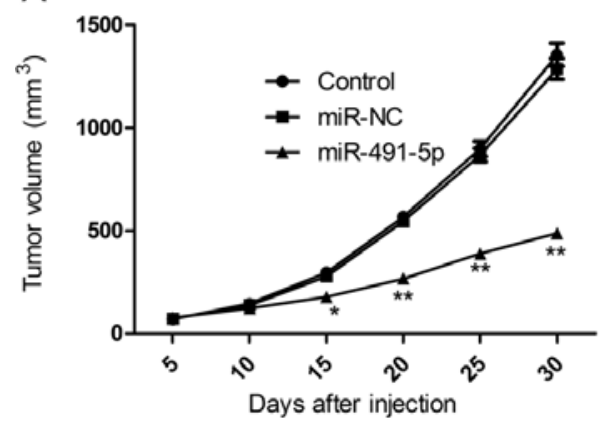

B

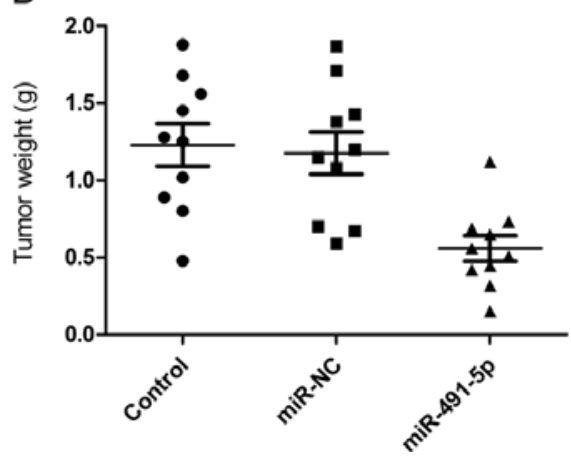

C

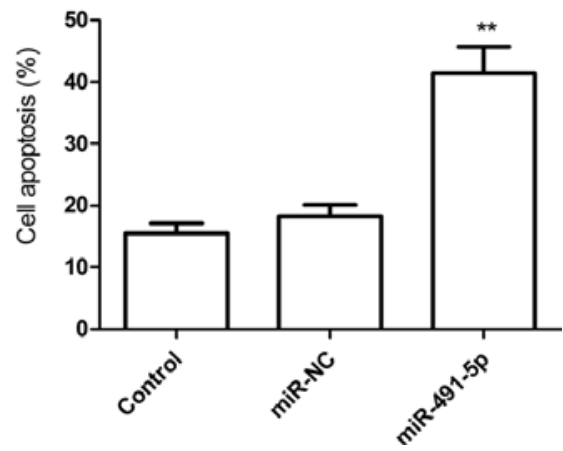

Figure 6. miR-491-5p upregulation suppresses cervical cancer HeLa xenograft growth. (A) Growth curves of tumor volumes in xenografts of nude mice were established based on the tumor volume measured every five days until 30 days. (B) The weight of tumor tissues of different groups (n=10) was measured. (C) Cell apoptosis of tumor tissue was determined by TUNEL assay. ${ }^{* *} \mathrm{P}<0.01$, vs. miR-NC group.

survival in patients with cervical cancer. The identification of miRNAs has broadened our understanding of the mechanisms involved in tumorigenesis. In the present study, we reported that miR-491-5p expression was downregulated in human cervical cancer tissues and cervical cell lines compared to the corresponding adjacent normal tissue and human normal cervical squamous cells (NCSCs), respectively, and its downregulation was associated with FIGO stage and lymph node metastasis. Moreover, the overexpression of miR-491-5p suppressed tumor growth of cervical cancer in vitro and in vivo. These findings provide insights into cervical cancer research and therapeutic strategies for malignant cervical cancer.

Accumulating evidence has shown that miRNAs functions as either tumorigenic or tumor-suppressing genes (4, 6, 8 and 24). miR-491 functions as a tumor-suppressor gene in vitro, and is downregulated in several types of cancer (11-16). Furthermore, miR-491-5p, a mature form of miR-491, has been found to suppress the growth of several cancer cells by multiple target genes. For example, Huang et al found that miR-491-5p overexpression in invasive OSCC cells suppressed their migratory behavior in vitro and lung metastatic behavior in vivo by targeting the G-protein-coupled receptor kinaseinteracting protein 1 (GIT1) gene (11). Guo et al reported that the overexpression of miR491-5p in the SW1990 pancreatic cancer cell line markedly reduced cell growth and induced cell apoptosis through a mitochondrial-mediated intrinsic pathway by targeting TP53 and Bcl-2 (12). Denoyelle et al showed that miR-491-5p efficiently induces apoptosis in the IGROV1-R10 ovarian cancer cell line by directly inhibiting BCL-XL expression and inducing BIM accumulation in its dephosphorylated form by directly targeting the epidermal growth factor receptor (EGFR) (13). Li et al demonstrated that miR-491-5p inhibited glioblastoma cell proliferation and invasion by directly targeting EGFR, CDK6 and Bcl-xL (14). In the present study, we found that overexpression of miR491-5p inhibited cervical cell proliferation, migration and invasion in vitro, and suppressed tumor growth in vivo by targeting hTERT.

Telomerase activity is crucial to maintain the integrity of the replicating tumor cell and establish immortality, which is required for the survival of the majority of tumor cells $(25,26)$. It has been reported that telomerase expression was upregulated in more than $90 \%$ of malignant tumors including cervical cancer, while is absent in most normal somatic tissues (27-29). Human telomerase reverse transcriptase (hTERT), the catalytic subunit of telomerase, is a core component of the telomerase holoenzyme, and regulates telomerase activity $(23,30)$. It has been shown that hTERT is important in cancer tumorigenesis, growth, migration and invasion $(31,32)$. Recent findings have shown that the suppression of hTERT expression by siRNA inhibited cervical cancer cell growth in vitro and in vivo (23). However, few studies have focused on the correlation between miRNA and telomerase in cancer cells. Recent findings have shown that hTERT is a target gene of miR-138 in thyroid carcinoma cell lines (33), of miR-21 in glioblastoma (GBM) carcinogenesis (34), and of miR-1207-5p and miR-1266 in gastric cancer (35). Using several algorithms, we identified hTERT as potential targets of miR-491-5p. The luciferase activity assay and point mutation analysis demonstrated that the downregulation of hTERT was mediated by miR-491-5p through the hTERT-3'-UTR. Expression of hTERT proteins was also significantly downregulated in miR-491-5p-overexpressing HeLa cells. These results suggest that hTERT is a novel target gene of miR-491-5p.

The PI3K/Akt signaling pathway plays a crucial role in cancer cell proliferation during the development of cervical cancer, and downstream effectors of PI3K/Akt signaling were promising targets for cervical cancer therapy $(23,36)$. Recent findings showed that miR-491-5p regulated the PI3K/AKT signaling pathway in pancreatic cancer and ovarian cells $(12,13)$. Consistent with those results, the present study shows that miR-491-5p inhibited p-PI3K and p-AKT expression in cervical cancer cells, without total PI3K and AKT protein expression. In addition, a previous study showed that the downregulation of hTERT inhibited the PI3K/AKT signaling pathway (23). These studies suggest that miR-491-5p suppressed cervical cancer growth via the PI3K/AKT signaling pathway by targeting hTERT.

Collectively, the present study provides evidence that miR-491-5p is downregulated in cervical cancer tissues and cervical cancer cell lines. Additionally, its downregulation correlated with lymph node metastasis, poorly differentiated tumors and advanced FIGO stage. miR-338-3p functions as a tumor suppressor to inhibit cell proliferation, migration and invasion, and to induce cell apoptosis in vitro, as well as to suppress tumor growth in a nude mouse model. hTERT was 
identified as a novel target gene of miR-491-5p. In addition, we found that the overexpression of miR-491-5p decreased hTERT expression and inhibited the PI3K/AKT signaling pathways. These results suggest that miR-491-5p may be a novel tumor suppressor that blocks the growth of cervical cancer cells through PI3K/AKT signaling pathways by targeting hTERT.

\section{Acknowledgements}

The present study was supported by the Scientific Research Project of Jilin Provincial Bureau of Health (2013ZC005).

\section{References}

1. Siegel R, Naishadham D and Jemal A: Cancer statistics, 2012. CA Cancer J Clin 62: 10-29, 2012.

2. Duenas-Gonzalez A, Serrano-Olvera A, Cetina L and Coronel J: New molecular targets against cervical cancer. Int J Womens Health 6: 1023-1031, 2014.

3. de Freitas AC, Gomes Leitão Mda C and Coimbra EC: Prospects of molecularly-targeted therapies for cervical cancer treatment. Curr Drug Targets 16: 77-91, 2015.

4. Cummins JM and Velculescu VE: Implications of micro-RNA profiling for cancer diagnosis. Oncogene 25: 6220-6227, 2006.

5. Miska EA: How microRNAs control cell division, differentiation and death. Curr Opin Genet Dev 15: 563-568, 2005.

6. Croce CM and Calin GA: miRNAs, cancer, and stem cell division. Cell 122: 6-7, 2005.

7. Kozomara A and Griffiths-Jones S: miRBase: Annotating high confidence microRNAs using deep sequencing data. Nucleic Acids Res 42: D68-D73, 2014.

8. He L, Thomson JM, Hemann MT, Hernando-Monge E, Mu D Goodson S, Powers S, Cordon-Cardo C, Lowe SW, Hannon GJ et al: A microRNA polycistron as a potential human oncogene Nature 435: 828-833, 2005.

9. Munker R and Calin GA: MicroRNA profiling in cancer. Clin Sci 121: 141-158, 2011.

10. Li C, Feng Y, Coukos G and Zhang L. Therapeutic microRNA strategies in human cancer. AAPS J 11: 747-757, 2009.

11. Huang WC, Chan SH, Jang TH, Chang JW, Ko YC, Yen TC, Chiang SL, Chiang WF, Shieh TY, Liao CT, et al: miRNA-491-5p and GIT1 serve as modulators and biomarkers for oral squamous cell carcinoma invasion and metastasis. Cancer Res 74: 751-764, 2014.

12. Guo R, Wang Y, Shi WY, Liu B, Hou SQ and Liu L: MicroRNA miR-491-5p targeting both TP53 and Bcl-XL induces cell apoptosis in SW1990 pancreatic cancer cells through mitochondria mediated pathway. Molecules 17: 14733-14747, 2012.

13. Denoyelle $C$, Lambert $B$, Meryet-Figuière $M$, Vigneron $N$, Brotin E, Lecerf C, Abeilard E, Giffard F, Louis MH, Gauduchon $\mathrm{P}$, et al: $\mathrm{miR}-491-5 \mathrm{p}-$ induced apoptosis in ovarian carcinoma depends on the direct inhibition of both BCL- $\mathrm{X}_{\mathrm{L}}$ and EGFR leading to BIM activation. Cell Death Dis 5: e1445, 2014.

14. Li X, Liu Y, Granberg KJ, Wang Q, Moore LM, Ji P, Gumin J, Sulman EP, Calin GA, Haapasalo H, et al: Two mature products of $M I R-491$ coordinate to suppress key cancer hallmarks in glioblastoma. Oncogene 34: 1619-1628, 2015.

15. Leivonen SK, Sahlberg KK, Mäkelä R, Due EU, Kallioniemi O, Børresen-Dale AL and Perälä M: High-throughput screens identify microRNAs essential for HER2 positive breast cancer cell growth. Mol Oncol 8: 93-104, 2014.

16. Zhou Y, Li Y, Ye J, Jiang R, Yan H, Yang X, Liu Q and Zhang J: MicroRNA-491 is involved in metastasis of hepatocellular carcinoma by inhibitions of matrix metalloproteinase and epithelial to mesenchymal transition. Liver Int 33: 1271-1280, 2013.
17. Nakano H, Miyazawa T, Kinoshita K, Yamada Y and Yoshida T: Functional screening identifies a microRNA, miR-491 that induces apoptosis by targeting $\mathrm{Bcl}-\mathrm{X}_{\mathrm{L}}$ in colorectal cancer cells. Int J Cancer 127: 1072-1080, 2010

18. Yan W, Zhang W, Sun L, Liu Y, You G, Wang Y, Kang C, You Y and Jiang T: Identification of MMP-9 specific microRNA expression profile as potential targets of anti-invasion therapy in glioblastoma multiforme. Brain Res 1411: 108-115, 2011.

19. Rutnam ZJ and Yang BB: The non-coding 3' UTR of CD44 induces metastasis by regulating extracellular matrix functions. J Cell Sci 125: 2075-2085, 2012.

20. Hacker NF: Revised FIGO staging for carcinoma of the vulva. Int J Gynaecol Obstet 105: 105-106, 2009.

21. Libra M, Scalisi A, Vella N, Clementi S, Sorio R, Stivala F, Spandidos DA and Mazzarino C: Uterine cervical carcinoma: Role of matrix metalloproteinases (Review). Int J Oncol 34: 897-903, 2009.

22. Ghosh A, Moirangthem A, Dalui R, Ghosh T, Bandyopadhyay A, Dasgupta A, Banerjee U, Jana N and Basu A: Expression of matrix metalloproteinase-2 and 9 in cervical intraepithelial neoplasia and cervical carcinoma among different age groups of premenopausal and postmenopausal women. J Cancer Res Clin Oncol 140: 1585-1593, 2014.

23. Shi YA, Zhao Q, Zhang LH, Du W, Wang XY, He X, Wu S and Li YL: Knockdown of hTERT by siRNA inhibits cervical cancer cell growth in vitro and in vivo. Int J Oncol 45: 1216-1224, 2014.

24. Ventura A and Jacks T: MicroRNAs and cancer: Short RNAs go a long way. Cell 136: 586-591, 2009.

25. Liu JP, Chen W, Schwarer AP and Li H: Telomerase in cancer immunotherapy. Biochim Biophys Acta 1805: 35-42, 2010.

6. Chen H, Li Y and Tollefsbol TO: Strategies targeting telomerase inhibition. Mol Biotechnol 41: 194-199, 2009.

27. Shay JW and Bacchetti S: A survey of telomerase activity in human cancer. Eur J Cancer 33: 787-791, 1997.

28. Gül I, Dündar O, Bodur S, Tunca Y and Tütüncü L: The status of telomerase enzyme activity in benign and malignant gynaecologic pathologies. Balkan Med J 30: 287-292, 2013.

29. Rosa MI, Medeiros LR, Bozzetti MC, Fachel J, Wendland E, Zanini RR, Moraes AB and Rosa DD: Accuracy of telomerase in cervical lesions: A systematic review. Int J Gynecol Cancer 17: 1205-1214, 2007.

30. Zhang W and Xing L: RNAi gene therapy of SiHa cells via targeting human TERT induces growth inhibition and enhances radiosensitivity. Int J Oncol 43: 1228-1234, 2013.

31. Cifuentes-Rojas C and Shippen DE: Telomerase regulation. Mutat Res 730: 20-27, 2012

32. Noël JF and Wellinger RJ: Exposing secrets of telomere-telomerase encounters. Cell 150: 453-454, 2012.

33. Mitomo S, Maesawa C, Ogasawara S, Iwaya T, Shibazaki M, Yashima-Abo A, Kotani K, Oikawa H, Sakurai E, Izutsu N, et al: Downregulation of miR-138 is associated with overexpression of human telomerase reverse transcriptase protein in human anaplastic thyroid carcinoma cell lines. Cancer Sci 99: 280-286, 2008.

34. Wang YY, Sun G, Luo H, Wang XF, Lan FM, Yue X, Fu LS, Pu PY, Kang CS, Liu N, et al: miR-21 modulates hTERT through a STAT3-dependent manner on glioblastoma cell growth. CNS Neurosci Ther 18: 722-728, 2012

35. Chen L, Lü MH, Zhang D, Hao NB, Fan YH, Wu YY, Wang SM, Xie R, Fang DC, Zhang H, et al: miR-1207-5p and miR-1266 suppress gastric cancer growth and invasion by targeting telomerase reverse transcriptase. Cell Death Dis 5: e1034, 2014.

36. Wu J, Chen $\mathrm{C}$ and Zhao KN: Phosphatidylinositol 3-kinase signaling as a therapeutic target for cervical cancer. Curr Cancer Drug Targets 13: 143-156, 2013. 